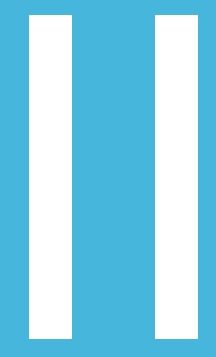

\title{
MODELO DE APROXIMACIÓN PARA LA VALUACIÓN DE EMPRESAS DEL CORPORATIVO ADVENTISTA
}

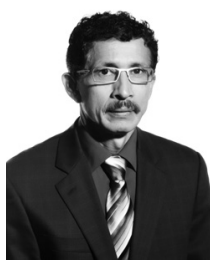

\section{Pedro Gonzales}

Licenciado en Contabilidad por la Universidad Peruana Unión. Doctor en Administración por la Universidad Nacional Federico Villarreal. Actualmente se desempeña como docente de posgrado de la Universidad de Montemorelos. Asimismo, realizó actividades de docencia en diversos posgrados organizados por dicha universidad. Ha publicado numerosos artículos en revistas nacionales y extranjeras. 


\section{Resumen}

El objetivo de esta investigación es generar un aporte al establecimiento de un modelo de valuación para las empresas del corporativo adventista en la región de Latinoamérica, partiendo desde un enfoque bíblico de las finanzas - que constituyen sólidos antecedentes de los fundamentos y principios básicos de las finanzas empresariales de nuestro tiempo - con el propósito de contribuir a una mejor gestión financiera en dichas empresas. Las finanzas modernas basan su accionar en principios fundamentales del valor del dinero, el riesgo, el rendimiento, el costo de capital, los flujos de efectivo etc. Algunos de estos principios tienen antecedentes en el registro bíblico, y constituyen elementos claves para la creación de un modelo financiero que nos permiten una aproximación a la valoración de las instituciones empresariales adventistas. El estudio tiene dos fases, la primera comprende el diseño del modelo teórico y su difusión, y la segunda fase la contrastación del modelo con la información real de las empresas del corporativo adventista durante los últimos cinco años.

Palabras clave: Valuación de empresas, valor del dinero, riesgo, rendimiento, costo de capital, flujo de efectivo.

\section{Abstract}

The objective of this research is to generate a contribution to the establishment of a valuation model for corporate companies Adventist in the Latin American region, starting from a biblical approach to finance - which constitute a solid background in the fundamentals and basic principles of finance business of our time - in order to contribute to better financial management in these companies. Modern finance their actions based on fundamental principles of value for money, risk, performance, cost of capital, cash flows etc. Some of these principles have a history in the biblical record, and are key to the creation of a financial model that allows us to approximate the value of Adventist business institutions elements. The study has two phases, the first comprising the design of the theoretical model and its diffusion and the second phase testing of the model with real information Adventist corporate companies over the past five years.

Keywords: Valuation of companies, value of money, risk, performance, cost of capital, cash flow. 


\section{Introducción}

La Iglesia Adventista del Séptimo Día posee una red de instituciones a nivel mundial entre las que se encuentran fábricas de alimentos, sanatorios, clínicas, colegios, universidades, instituciones de salud etc. Muchas de ellas operan de manera empresarial en mercados muy competitivos a nivel nacional e internacional. Esta dimensión de la actividad empresarial de la Iglesia hace necesario la adopción de nuevos enfoques en la gestión financiera, en cuanto al análisis del valor económico de dichas instituciones.

El análisis del valor económico se ha convertido en un elemento fundamental para la toma de decisiones financieras en las empresas (financiación, inversión, retribución al capital, etc.). La determinación del valor de la empresa es el paso previo antes de decidir si seguir en el negocio, vender, fusionarse, crecer o comprar otras empresas.

Es evidente que los directivos de una empresa, más tarde o más temprano y por la razón que fuere, deben preguntarse sobre cuál es el valor de su negocio. A menudo la respuesta no es fácil. La contabilidad no siempre ayuda ¿Cuánto vale la empresa? ¿Cual es el valor de participación? ¿Cuál es el valor máximo que se debería pagar por ella? ¿Cuál es el valor mínimo que se deberá aceptar? Son algunas de las cuestiones que propietarios, accionistas, compradores, inversionistas y agentes económicos tienen que resolver. La respuesta, lejos de estar al alcance de los interesados, se asienta muchas veces en procesos técnicos externos muy laboriosos y casi siempre bastante costosos. Sin embargo, la realidad financiera y tecnológica nos muestra que existen técnicas que permiten realizar dicho proceso de valoración de una manera más fluida, eficiente (coste económico) y no por ello menos rigurosos.

En los ámbitos contable y financiero, el tema de la valuación de empresas juega un papel fundamental para tomar decisiones de: adquisiciones, fusiones o ventas de empresas, conocer la situación del patrimonio, verificar la gestión llevada a cabo por los directivos, estudiar la capacidad de deuda, reestructuración de capital, decisiones estratégicas acerca de la continuidad de la empresa, establecimiento de acuerdos de compra-venta, capitalización de una empresa, valoración de activos intangibles (fondo de comercio), obtención de líneas de financiación, valoraciones patrimoniales, valoraciones fiscales, suspensiones de pagos, etc.

La valoración de una empresa también puede ayudar a responder preguntas tales como:

- ¿Cuál ha sido la rentabilidad sobre la inversión de mi negocio? 
- ¿ ¿Qué se puede hacer para mejorar esta rentabilidad y crear riqueza?

\section{Fundamentos teóricos de valoración de empresas Antecedentes bíblicos}

La Biblia muestra referencias muy puntuales y claras con respecto a los elementos claves que conforman un modelo para valorar empresas, en el libro de Mateo hay referencias y expresiones financieras referidas a principios fundamentales de las finanzas, allí encontramos alusiones al valor temporal del dinero, encontramos también una apreciación de lo que es el riesgo, el rendimiento y el costo de oportunidad, del mismo modo, en el libro de Levítico podemos apreciar con mayor claridad un sistema básico de valoración de bienes o activos que hacía el sacerdote conforme a las instrucciones que Dios había establecido. Veamos con mayor detalle estos elementos en el registro bíblico.

\section{El valor del dinero en el tiempo}

- ¿ ¿ por qué no depositaste mi dinero en el banco? Al menos hubiera podido obtener algún interés de él. Mat. 25:27, NTV )

- ... deberías haber llevado mi dinero al banco...( Mat. 25:27, DHH)

El concepto del valor temporal del dinero se refiere al hecho de que un dólar en la mano hoy, vale más que un dólar en algún momento futuro. Pero, ¿cómo puede ser eso? Un dólar sigue siendo un dólar, en el presente y en el futuro. Sí, pero un dólar en la mano hoy en día, se puede invertir en una cuenta que genere intereses u otro tipo de rendimientos que crecen en valor con el tiempo. Esto explica, en parte, por qué el valor del dinero está relacionado con el tiempo. En el caso de la parábola, notamos que el tercero de los empleados descuidó ese aspecto y guardó el dinero bajo tierra, con la idea que la persona que le encargó esos recursos era un hombre duro, que cosechaba donde no sembraba y recogía donde no esparcía, sin embargo, tenía el deber (deberías haber llevado) de lograr por lo menos los intereses. Esa expresión "al menos" significa que era lo mínimo que se esperaba que hiciera, al evaluar las alternativas disponibles, esto lleva a considerar que el valor temporal del dinero está relacionado con otro concepto llamado costo de oportunidad. 


\section{Costo de oportunidad}

El costo de oportunidad de capital de la empresa es la rentabilidad mínima exigida por los inversionistas de un proyecto o empresa, la cual se emplea como tasa de descuento para actualizar los flujos de las empresas, los autores Polimeni, Fabozzi y Adekberg (1998) definen el costo de oportunidad de la siguiente manera: cuando se toma una decisión para empeñarse en determinada alternativa, se abandonan los beneficios de otras opciones. Los beneficios perdidos al descartar la siguiente mejor alternativa son los costos de oportunidad de la acción escogida.

Un análisis más amplio del incidente bíblico nos lleva a considerar que el costo de oportunidad para el empleado improductivo era la tasa de interés que pagaba el banco en aquel entonces, tasa que según se denota en el relato bíblico era, al menos, la alternativa menos riesgosa. Ahora bien, si trasladamos a este tiempo este incidente, tendríamos como expresión equivalente lo que hoy llamamos "la tasa libre de riesgo" que se ofrece en el sistema financiero, para inversiones en títulos valores de los estados o gobiernos, o bonos del tesoro que están respaldados por los países que los emiten. Esta es una tasa de referencia para inversionistas que cuidan minimizar el riesgo de su inversión, podríamos decir parafraseando, para este tiempo, la declaración del inversionista bíblico: "debiste haber logrado por lo menos la tasa libre de riesgo. Un costo de oportunidad según Thompson y Antezana (2008) requiere que abandonemos un beneficio, se da principalmente porque existen dos o más alternativas de inversión para ganar dinero, entonces se busca la solución más inteligente, "más rentable".

\section{Riesgo}

"Tenía miedo de perder su dinero, así que lo escondí en la tierra. Mire, aquí está su dinero de vuelta". (Mat. 25:25, NTV)

Uno de los aspectos importantes que hay que evaluar en cualquier decisión de inversión, es el riesgo. García (1999) nos refiere acerca del origen de esta palabra que, en muchos de los idiomas modernos, tiene la misma raíz - la española "riesgo", la francesa "risque", la italiana "rischio", la inglesa "risk", proviene del latin risicare. En la antigüedad llamaban risicare (lat) a la capacidad de navegar al lado de un arrecife o roca. En realidad tiene un significado negativo relacionado con el peligro, daño, siniestro o pérdida. Sin embargo, riesgo es parte inevitable de los procesos de toma de decisiones en general y de los procesos de inversión en particular. El riesgo ha sido, desde antaño, una variable ineludible en toda decisión de inversión empresarial. 
En el relato bíblico que estamos analizando ¿que alegó como pretexto el administrador improductivo? ¡Señor tuve miedo..!. Esta simple respuesta presupone una acepción de lo que es el riesgo empresarial; según Cooper y Chapman (1987) riesgo es la exposición a la posibilidad de pérdidas económicas, financieras o de otros perjuicios como consecuencia de la incertidumbre que origina el llevar a cabo una determinada acción. El miedo es algo natural en el ser humano, y es de suponer que los empleados que lograron altos rendimientos también experimentaron ese temor, la diferencia es que el temor no los paralizó.

Un mayor análisis de este incidente, desde el punto de vista financiero, nos permite inferir lo siguiente: a). Que el riesgo se puede evitar no haciendo nada -inversión con riesgo cero- como fue el caso del tercer "empleado o administrador". Con la salvedad que no correr un riesgo es de por sí un riesgo. b). La inversión debe rendir al menos una tasa libre de riesgo, -inversiones con riesgo mínimo- como es el rendimiento que se obtiene al depositar el dinero en el Banco. Lo que se pretende con ello es evitar el impacto de la devaluación. c). Los inversionistas que arriesgan pueden obtener altas rentabilidades, tal fue el caso de los dos primeros empleados.

\section{El flujo de efectivo descontado}

En los versículos 1, 16, 17, 18 del capítulo 27 del libro de Levítico, la versión Dios Habla Hoy (DHH) declara: "El Señor se dirigió a Moisés y le dijo: "Si alguien consagra al Señor una parte de su terreno, el valor del terreno se calculará según lo que pueda producir, a razón de cincuenta monedas de plata por cada doscientos veinte litros de cebada. Si consagra el terreno a partir del año de liberación, quedará en pie el valor establecido; pero si lo consagra después del año de liberación, el sacerdote hará el cálculo de la plata que se debe dar, descontando, del valor calculado, la cantidad que corresponda a los años que restan hasta el año de liberación.

La valuación, mediante flujos de efectivo descontado, era realizada desde los tiempos bíblicos, el sacerdote era el indicado de llevar adelante este proceso de cálculo, bajo ciertas pautas dadas por Dios a través de Moisés. Este proceso, según el relato bíblico, consistía en establecer un valor estimado de acuerdo a los flujos futuros que generara el terreno, en este caso el valor del terreno se calculaba según "lo que podía producir", a razón de cincuenta monedas de plata por cada doscientos veinte litros de cebada, el sacerdote hacía el cálculo de la plata que se debía dar, descontando del valor calculado la cantidad que correspondía a los años que restaban, hasta el año de liberación o del jubileo. 
De acuerdo con Saavedra (2007, p. 104):

"El modelo de flujo de efectivo disponible considera que el valor de la empresa en marcha estará dado por el flujo de efectivo libre disponible que se espera que genere en el futuro, descontado a una tasa que refleje los riesgos del negocio".

Como podemos apreciar, las referencias bíblicas que hemos analizado brevemente nos muestran una ilustración de valuación con ciertas características de ese tiempo y contexto, esas técnicas hoy con algunas innovaciones y mayores recursos tecnológicos y financieros constituyen hoy los fundamentos para la toma de decisiones financieras.

Estos mismos elementos son los que estudiaremos para la creación de un modelo de valoración de las empresas del corporativo adventista. El modelo es de aplicación a las empresas más competitivas y estables que tiene la Iglesia adventista, que se mantienen bajo un sistema administrativo común, se orientan por una sola misión con los mismos principios administrativos y filosóficos de la organización.

\section{Diferencia entre valor y precio}

Es importante distinguir entre valor y precio, dado que ambas expresiones no significan lo mismo, Molina y Trenor (2003) definen en forma amplia la valoración "como el proceso mediante el cual se busca la cuantificación de los elementos que constituyen el patrimonio de una empresa, su actividad, su potencialidad o cualquier otra característica de la misma susceptible de ser valorada. Los autores señalan también que "al valorar una empresa se pretende determinar el intervalo de valores razonables dentro del cual estará incluido el valor definitivo. Se trata de determinar el valor intrínseco de un negocio y no su valor de mercado ni, por supuesto, su precio. El valor no es un hecho sino que, debido a su subjetividad, podríamos considerarlo como una opinión. Se debe partir de la idea de que el valor es solamente una posibilidad, mientras que el precio es una realidad".

El valor puede ser distinto para diversos compradores y para el vendedor, en tanto que el precio es la cantidad que acuerdan, tanto vendedor como comprador, al concretar una operación de compra-venta de una empresa. Fernandez, (2001) a través de un ejemplo que exponemos aclara el sentido de esta diferencia. Al respecto señala: suponiendo que una empresa extranjera, muy avanzada tecnológicamente, quisiese comprar una empresa muy conocida en el mercado nacional con el objetivo de conquistar tal mercado, valorará solamente la marca de la empresa nacional. Poco importarán al efecto el 
valor de sus maquinarias o instalaciones, ya que lo avanzado tecnológicamente de la empresa extranjera hará que a esta no le interesen tales bienes. Por el contrario, a la empresa nacional le interesará valuar muy bien sus recursos materiales, dado su posición en el mercado.

La empresa extranjera deberá determinar el valor máximo que estaría dispuesta a pagar por lo que le aportaría la empresa nacional. Desde el punto de vista de esta última interesará conocer el valor mínimo al que debería aceptar la operación. Son dos cifras que se confrontan en una negociación, al final de la cual se acordará un precio que, generalmente, suele estar en algún punto intermedio de ambas.

\section{Métodos de valoración}

Existe una diversidad de métodos de valoración que se han desarrollado a lo largo de muchos años, en distintas circunstancias y con diversos grados de acierto. Según e-Valora, empresa especializada en temas de valoración, en su manual de valoración menciona que cada método de valoración tiene ventajas y desventajas en su aplicación, y han de ser utilizados con cuidado, ya que cada uno de ellos será más apropiado para determinadas circunstancias. Manual de la compañía e - Valora (2011)

Figura 1. Métodos de valoración de empresas de Empresa de Consultores e-Valora

\section{Modelos básicos de valoración de empresas}

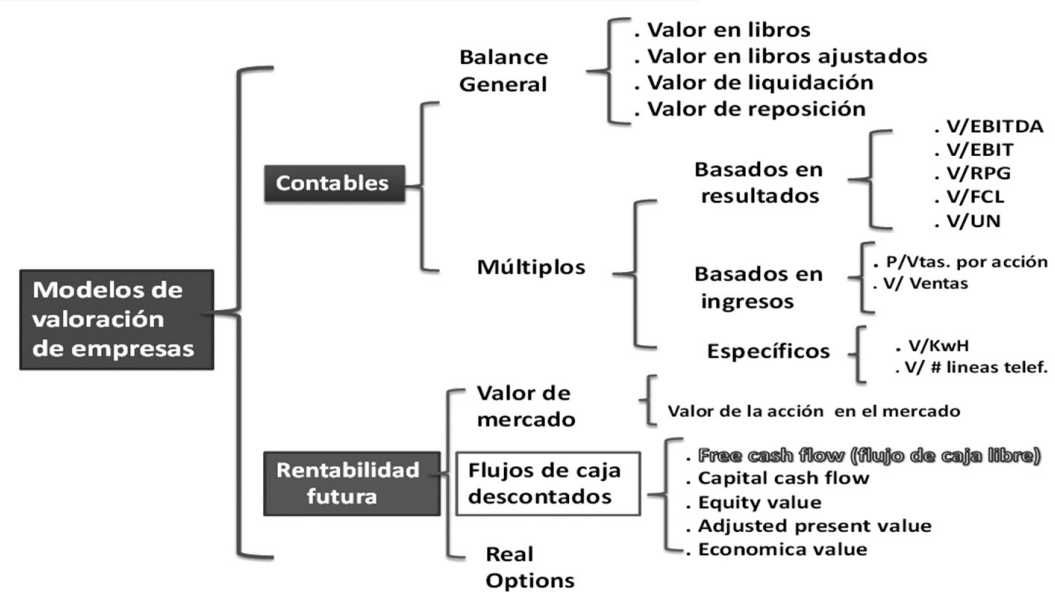




\section{Métodos basados en el balance patrimonial}

Estos métodos intentan calcular el valor de la empresa en base a la estimación del valor de su patrimonio. Son los que tradicionalmente consideran que el valor de una empresa radica esencialmente en su balance. Su visión es estática y no tienen en cuenta la evolución futura de la empresa, el valor temporal del dinero, ni otros factores como: la situación del sector, problemas en relación con los recursos humanos, en relación con la organización, etc. situaciones que no se reflejan en los estados contables. Entre estos métodos se pueden citar: valor contable, valor contable ajustado, valor de liquidación y valor sustancial.

\section{Métodos basados en cuenta de resultados}

Se diferencia de los anteriores porque se basan en la cuenta de resultados, toman magnitudes que extraen de los beneficios, de las ventas o de otro indicador. Utilizan múltiplos para su cálculo, tal es el caso de los métodos basados en el PER, donde el precio de la acción es un múltiplo del beneficio. El PER de una acción indica el múltiplo del beneficio por acción que se paga en la bolsa. Entre estos métodos se pueden citar: el valor de los beneficios: PER, el valor de los dividendos, el múltiplo de las ventas, otros múltiplos y múltiplos utilizados para valorar empresas en Internet.

\section{Fondo de Comercio (Goodwill)}

El fondo de comercio es el mayor valor que tiene una empresa por encima del valor contable o valor contable ajustado. Intenta representar el valor de los elementos inmateriales de la empresa que, muchas veces, no aparece reflejado en el balance, pero que le proporcionan ventajas competitivas (ejemplo: cartera de clientes, liderazgo sectorial, marcas, alianzas estratégicas, etc.). Una valoración correcta, bajo este método, requerirá añadir al activo neto estos inmateriales. El problema se presenta en el momento de calcular su valor, ya que existen diferentes metodologías.

Estos métodos parten de una visión mixta: valoran en forma estática a los activos y lo valoran en forma dinámica al tratar de cuantificar el valor que generará la empresa en el futuro. El autor sostiene que, en su determinación, existe mucha arbitrariedad y, en consecuencia, no comparte su utilización en la práctica. Agrega en ellos no se puede encontrar "mucha ciencia". 
Entre estos métodos se pueden citar: el Método de valoración “Clásico", el Método simplificado de la "renta abreviada del goodwill" o el método de la UEC (Unión de Expertos Contables Europeos") simplificado, el Método de la UEC propiamente dicho, el Método indirecto o el método de "los prácticos", el Método anglosajón o el método directo, el Método de compra de resultados anuales y el Método de la tasa con riesgo y de la tasa sin riesgo.

\section{Método de flujos de caja descontados}

Estos métodos intentan determinar el valor de la empresa en función de la estimación de los flujos de dinero -cash flows- que generará en el futuro, descontándolos a una tasa de descuento relacionada con el riesgo de dichos flujos. En otras palabras, es obtener el valor actual de los flujos de fondos esperados. Para el autor, es el método más apropiado para valorar una empresa. Requieren un pronóstico detallado y minucioso para cada período, de cada una de las partidas financieras relacionadas a la generación de los cash flows de la empresa, como: cobro de ventas, pagos, etc. Funcionaría como un presupuesto de tesorería. El inconveniente se encuentra en la elección de la tasa de descuento apropiada, percepciones sobre el sector y la empresa, etc.

Este método de valoración de empresas es el más recomendado en la mayoría de los casos, la estimación de los flujos de caja que deben ser generados en el futuro y descontados a una tasa coincidente con el riesgo de los mismos. Según la empresa e-Valora, estos métodos identifican el valor absoluto de un negocio, por lo que no se necesita ninguna comparación con compañías similares y permite tener en cuenta todos los factores de éxito explícitamente. El método se expresa matemáticamente mediante la siguiente relación:

Valor $=\frac{F C L_{1}}{(1+T D)^{1}}+\frac{F C L_{2}}{(1+T D)^{2}}+\ldots+\frac{F C L_{n}+V R_{n}}{(1+T D)^{n}}$

Dónde:

$$
\begin{aligned}
& \text { FCF = Flujo de Caja Libre para la Empresa } \\
& T D=\text { Tasa de Descuento } \quad \text { VR = Valor Residual }
\end{aligned}
$$

Así, el método consta de cuatro elementos básicos:

1- Flujo de Caja Libre para la Empresa (FCFF)

2- Tasa de Descuento/ Coste de Capital (TD)

3- Horizonte temporal (n)

4- Valor Residual (VT) o Terminal 


\section{Metodología del modelo propuesto}

A continuación describimos cómo se aplica este método. Los elementos principales para la valuación de una empresa están dados por la existencia de ciertos principios financieros que se vieron al inicio de este trabajo, los mismos que también fueron analizados a partir de sus antecedentes bíblicos. El elemento integrador de todos estos elementos es, sin duda, el flujo de caja descontado que se apoya en el costo de oportunidad, el riesgo y la valoración de esos flujos de dinero a través del tiempo, para la determinación de un valor económico para la empresa. Este método está basado en la capacidad de generación de flujos futuros de la empresa. Así, el valor de la empresa se calculará estimando los flujos potenciales estimados.

Esta propuesta, que pretende un modelo de aproximación a la valoración de empresas adventistas, se apoya en el modelo de flujo de efectivo descontado, el que de acuerdo con Saavedra (2007, p.104):

El valor de una empresa en marcha estará dado por el flujo de efectivo libre disponible que se espera que genere en el futuro, descontando a una tasa que refleje los riesgos del negocio.

Del mismo modo, conforme lo indica Fernández (2008) los métodos basados en el descuento de flujos de fondos determinan el valor de las empresas a través de la estimación de los flujos de efectivo que generará en el futuro, para luego descontarlos a una tasa apropiada, según el riesgo de dichos flujos.

El modelo toma como punto de partida la determinación de los flujos de efectivo descontados, debemos estimar el flujo de caja libre de la empresa $(F C L)$, luego un modelo para la determinación de un costo de capital promedio ponderado para este tipo de empresas y, finalmente, así como de otras variables que intervienen en este proceso.

\section{El flujo de caja libre para la empresa (FCL)}

Representa los fondos que quedan disponibles para todos los proveedores de financiación de la empresa (accionistas y bancos u otros acreedores financieros).

Este método es ampliamente utilizado para estimar el valor de una empresa. Es un método dinámico que toma en consideración el valor del dinero a través del tiempo y que permite evaluar el efecto concreto de muchas 
variables en los rendimientos y comportamientos futuros. Las valoraciones por FCL pueden ser muy sensibles a pequeños cambios en algunos de los datos de partida, por lo que proporcionan al analista meticuloso una herramienta muy poderosa. Aunque se puede discutir que el método es complicado y subjetivo, no consideramos estas razones suficientes para rechazarlo, ya que lo mismo se puede decir de muchas técnicas de valoración.

Idéntico razonamiento se puede aplicar a la sensibilidad de los FCL sobre las hipótesis de crecimiento a largo plazo: mientras que este dato puede también afectar a otros métodos de valoración, en los FCL el efecto puede ser mitigado utilizando una tasa de crecimiento de valor añadido a largo plazo nula o muy baja, además realizando un estudio riguroso del valor que realmente queda en el negocio al final del período proyectado.

La formulación simplificada de este método puede expresarse como la sumatoria de los flujos de caja actualizados del período de previsión explícito, más el valor residual también actualizado:

Figura 2. Métodos de valoración mediante flujos de efectivo.

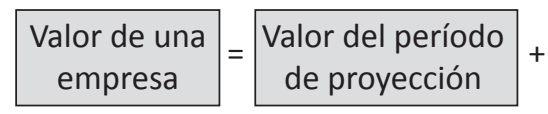

\section{Valor de una empresa}

VE: El valor actual de los flujos de caja libre

Según Panelati y otros, (2011) el valor de una empresa es el valor actual de los flujos de fondos estimados, se calcula con el flujo de caja libre descontado con la trema, la cantidad de períodos de proyección deben ser lo suficiente hasta tanto la empresa haya alcanzado un desarrollo estable de su actividad.

El valor del período de proyección vendría dado por:

$$
V E=\sum_{j=1}^{n} \frac{F C L_{j}}{T D+1}
$$

Siendo TD: la tasa de actualización o de descuento correspondiente al año j.

El flujo de caja libre (FCL) se calcula de acuerdo a lo siguiente:

$$
\mathrm{FCL}=\mathrm{U} . \mathrm{O}-\mathrm{I}+\mathrm{D}-\triangle \mathrm{CT}
$$


FCL : El valor de los flujos de efectivo disponible

U.O : Utilidad operativa

I : Impuestos

D : Depreciación y amortización

$\triangle C T$ : Incremento en el capital de trabajo

TD : Tasa de descuento

\section{El valor del período posterior a la proyección}

La proyección de los flujos, después del período de proyección, se comporta como una perpetuidad creciente. Sabemos que ese es el valor presente de una suma infinita, según el modelo de Castilla (2011). Este valor se calcula del siguiente modo:

$$
V R=\frac{F C L_{t+1}}{T D-g}
$$

$\mathrm{FCL}_{\mathrm{t}+1}$ : Flujo de efectivo libre del año siguiente al período explícito de pronóstico. " $\mathrm{g}$ ": $\quad$ Crecimiento esperado del FCL.

Ahora bien, estas expresiones pueden adoptar algunas variantes, ya que los flujos de los diferentes períodos pueden considerarse constantes o variables, el horizonte temporal finito o perpetuo, la tasa de actualización constante o variable anualmente. Para efectos de este modelo, se plantea que los flujos de caja libre son variables y se ajustarán a una tasa de crecimiento que va a depender del mejor resultado que ofrezca uno de los tres métodos estadísticos más usados para medir el crecimiento: los métodos geométrico, lineal y exponencial. Al calcular la tasa de crecimiento de los flujos de caja libre mediante los tres métodos estadísticos mencionados, se seleccionará, como el más óptimo, aquel que ofrezca mejor ajuste después de medir el error de cada uno de los resultados. El método a usar para medir el error o confiabilidad es el error absoluto medio porcentual (MAPE), también conocido como porcentaje de error medio absoluto (PEMA). Hemos seleccionado el criterio del error absoluto medio porcentual (MAPE), pues permite comparar diferentes modelos, dado que es independiente de la escala. 


\section{Cálculo de tasas de crecimiento de los flujos de caja y el patrimonio o capital contable}

\section{a) Método geométrico}

Este método resulta útil cuando solo se cuenta con los valores inicial y final de una serie, o cuando los valores observados estuvieran comprendidos en la línea recta trazada desde el valor inicial hasta el valor final. El cálculo de la tasa de crecimiento mediante el método geométrico resulta ser el más sencillo, se utilizan los valores inicial y final de la serie de acuerdo a la siguiente ecuación:

$$
r=\left[\frac{V f}{V i}\right]^{\frac{1}{n-1}}-1
$$

donde,

$r$ : es la tasa de crecimiento

Vf : es el valor final de la serie

$\mathrm{Vi}$ : es el valor inicial de la serie

$\mathrm{n}$ : es el número de observaciones

b) Método lineal

Este método implica el cálculo de una regresión lineal mediante Mínimos Cuadrados Ordinarios (MCO). Se asume que la relación existente entre una variable dependiente (las exportaciones o el área cosechada) y otra independiente (el tiempo) se puede describir a través de la ecuación de una recta:

$$
y_{t}=a+\beta x
$$

donde,

$y_{t}:$ son los flujos de caja libre

$a$ : es el intercepto

$\beta$ : es la pendiente de la recta

$x$ : es el tiempo

En este caso la tasa de crecimiento promedio anual se halla dividiendo el valor estimado de la pendiente entre la media aritmética de la variable dependiente: 


$$
r=\frac{\beta x}{y_{t}}
$$

c) Método exponencial

Este método implica el cálculo de una regresión exponencial mediante Mínimos Cuadrados Ordinarios (MCO). Se asume que la relación existente entre una variable dependiente (los flujos de caja) y otra independiente (el tiempo) se puede describir a través de la ecuación:

$$
y=a \cdot e^{b x}
$$

Esta ecuación de regresión exponencial, aunque no es lineal, es linealizable del tipo:

$y=a . b x$, tomando logaritmos ya que haciendo el cambio de variable $y=\log y$ tendremos que la función anterior nos generaría:

$$
y=\log y=\log (a \cdot b x)=\log a+x \log b
$$

Para poder aplicar el método necesitamos linealizar esta ecuación, para ello aplicamos logaritmos naturales:

$$
\ln y_{t}=\ln a+\beta \cdot x
$$

En este caso, la tasa de crecimiento promedio anual está representada por el valor estimado de la pendiente: $\quad r=\beta$

\section{Error Absoluto Medio Porcentual (MAPE)}

Este se calcula como el promedio de las diferencias absolutas entre los valores pronosticados y reales, y se expresa como porcentaje de los valores reales. Es decir, si hemos pronosticado $\mathrm{n}$ períodos y los valores reales corresponden a $\mathrm{n}$ periodos, MAPE se calcula como:

$$
M A P E=\frac{\sum \frac{y_{t-} \hat{y}_{t}}{y_{t}}}{n}
$$


donde,

$y_{t}$ : es el valor observado de la variable dependiente en el período $t$.

$\hat{y}_{t}$ : es el valor calculado de la variable dependiente, utilizando cualquiera de los modelos mencionados.

$\mathrm{n}$ : es el número de observaciones.

Al aplicar el MAPE a los resultados de los tres métodos estadísticos, tomando en cuenta que para calcular las tasas de crecimiento podremos elegir la tasa de aquel método que refleje el menor error estadístico.

\section{La trema o tasa de actualización}

Es necesario establecer una tasa que posibilite una homogenización de los flujos dados en distintos momentos de tiempo, la cuantía de la tasa depende tanto de la situación de la propia empresa como del entorno, de modo que cuando las perspectivas propias o del entorno sean desfavorables, el tipo de actualización será mayor para recoger el riesgo.

Para la aplicación de un modelo de valuación se requiere determinar una trema o tasa de descuento. La trema o tasa de actualización representa la tasa de rentabilidad que el inversionista exige a su inversión. Al ser una retribución por los recursos invertidos, la tasa de descuento mide el costo de oportunidad de dichos recursos, es decir cuánto deja de ganar el inversionista por colocar sus recursos en un proyecto-empresa. Para la determinación de esta tasa, existen tres modalidades:

a) La manera más práctica es utilizar la tasa de rentabilidad de proyectos o empresas similares de la actividad sectorial. Esto significa que si la empresa a evaluar es una empresa de heladería, en primer lugar, se debería establecer cuál es la rentabilidad histórica o rentabilidad esperada de empresas de heladería similares, y si ese dato no estuviera disponible, se recomienda utilizar la rentabilidad de la actividad del sector correspondiente. Lo recomendable es utilizar la tasa de una empresa de similar riesgo o un promedio de las tasas de varias empresas similares.

b) Una metodología más sofisticada es la aplicación de métodos o modelos de valoración de activos financieros que sistematizan la relación entre rentabilidad y riesgo. Estos son: el CAPM (Capital Asset Pricing Model ) y el WACC (weigted average costo of capital).

c) Una tercera opción es agregar un factor de corrección por riesgo a la tasa de mercado. 


\section{Selección de la tasa de descuento}

La tasa de descuento que se emplea para traer a valor presente los flujos de efectivo es el costo de capital promedio ponderado (WACC). El modelo del costo del capital promedio ponderado, conocido como WACC, es la tasa que debe utilizarse para descontar el flujo de fondos del activo o proyecto con financiamiento. En la práctica, la determinación de la tasa de descuento o coste del capital del activo financiero o proyecto de inversión se determina utilizando el CAPM para calcular el coste del capital propio - recursos propios- y el coste de la deuda, y combinando ambas tasas en un único coste se efectúa utilizando el WACC.

EI WACC representa el costo promedio de todas las fuentes de fondos (acciones y deuda), ponderado por el peso relativo de las mismas en la estructura de pasivos de la empresa (o proyecto). El coste del capital promedio ponderado se calcula del siguiente modo:

$$
\text { WACC }=\frac{V_{d}}{V_{d}+V_{e}} K_{d}(1-T)+\frac{V_{e}}{V_{d}+V_{e}} K_{e}
$$

Dónde:

$\mathrm{V}_{\mathrm{d}}=$ Valor de la deuda

$\mathrm{K}_{\mathrm{d}}=$ Costo de la deuda con costo

$\mathrm{V}_{\mathrm{e}}=$ Valor del capital

$\mathrm{K}_{\mathrm{e}}^{\mathrm{e}}=$ Costo del capital

$\mathrm{T}^{\mathrm{e}}=$ Tasa de impuestos

La estimación del costo de la deuda $\mathrm{K}_{d}$ se basa en las tasas de interés sobre la deuda de la empresa. Este costo de expresa después de impuestos, puesto que los pagos de intereses son fiscalmente deducibles. En el caso de las empresas adventistas, en algunos países existe el beneficio de la exoneración del impuesto a las ganancias por ser asociaciones sin fines de lucro, en este caso hay que eliminar el beneficio del escudo fiscal de la expresión del WACC:

$$
W A C C=\frac{V_{d}}{V_{d}+V_{e}} K_{d}+\frac{V_{e}}{V_{d}+V_{e}} K_{e}
$$

Para estimar el costo del capital propio $\mathrm{K}_{\mathrm{e}}$ se obtiene mediante el método del modelo de valuación de activos de capital, mejor conocido como CAPM. Este modelo, desarrollado inicialmente por el premio Nobel William 
Sharpe, parte sobre la base de que la tasa de rendimiento requerida de un inversor es igual a la tasa de rendimiento sin riesgo más una prima de riesgo, donde el único riesgo importante es el riesgo sistemático. Este nos indica cómo responde el rendimiento de la acción ante las variaciones sufridas en el rendimiento del mercado de valores, dicho riesgo se mide a través del coeficiente de volatilidad conocido como beta (ß).

\section{Determinación del Coeficiente Beta (ß)}

La beta (ß) mide la variación del rendimiento de un título con respecto a la variación del rendimiento del mercado, de tal manera que si ß toma un valor igual a uno, estará variando en la misma sintonía que el mercado de valores, pero si dicho valor fuese más pequeño, sus oscilaciones serían de menor tamaño que las del rendimiento del mercado. Ocurriendo lo contrario, si ß fuese mayor que uno, existen por lo menos tres alternativas para calcular un beta para una empresa:

- Utilización del CAPM usando la beta de una compañía comparable en Estados Unidos.

- Utilización de una beta comparable del sector de la empresa a nivel local, nacional o internacional.

- Utilización de betas contables.

Dumrauf (2003) plantea algunas inquietudes con respecto al método CAPM en relación a la estimación de la beta en un contexto de economías emergentes, donde el tamaño de mercado de capitales es reducido y las compañías son de capital cerrado, como es el caso de las empresas adventistas, ¿cómo valuar una empresa que no cotiza en bolsa y, por lo tanto, no se dispone de betas para calcular el CAPM.

La beta contable podría ser una respuesta a estos tipos de requerimientos. La beta contable de una empresa marca la sensibilidad de sus rendimientos contables al rendimiento promedio del mercado. El rendimiento contable puede medirse como utilidad contable (ya sea operativa o neta), rendimiento contable sobre el patrimonio (ROE), rendimiento contable sobre activos (ROA) u otra medida análoga. El rendimiento del mercado puede ser un índice de bolsa o un índice del mercado de raíz también contable (utilidad, ROE ó ROA promedio del mercado). El atractivo de una beta contable reside en el hecho de que la información contable es más abundante que la información del mercado accionario; esto es, aun en el caso de empresas de capital cerrado existentes, los datos contables son accesibles y podrían, en principio, ser utilizados para calcular una beta al estilo de las empresas que cotizan en bolsa. 
Por tal razón, es pertinente considerar la adaptación del modelo CAPM a este contexto utilizando betas contables en vez de betas bursátiles, aplicando la siguiente expresión:

$$
b_{i}=\frac{\operatorname{Cov}\left(x_{i}, x_{m}\right)}{\sigma^{2}\left(x_{m}\right)}
$$

donde:

$\mathrm{b}_{\mathrm{i}}=$ beta contable de la empresa $\mathrm{i}$

$\mathrm{x}_{\mathrm{i}}=$ resultado contable de la empresa $\mathrm{i}$

$\mathrm{x}_{\mathrm{m}}=$ resultado contable de la cartera del mercado

Las rentabilidades contables pueden variar, en algunos casos, si se utiliza las rentabilidades operacionales o patrimoniales de la empresa, haciéndose lo mismo con las rentabilidades operacionales de un conjunto de empresas (el mercado). Una vez definido la beta contable de la empresa, es posible aplicar el modelo CAPM adaptado, tomando en consideración que la tasa libre de riesgo es la tasa de bonos del tesoro que proporciona, en este caso, el Banco Central de Reserva.

\section{Conclusiones}

1. Existe un sólido fundamento bíblico para el establecimiento de un modelo de valoración para las empresas adventistas del corporativo adventista en Latinoamérica. Los elementos de un modelo de valoración como los flujos de efectivo, valor del dinero, costo de oportunidad y riesgo se evidencia claramente en el registro bíblico.

2. El método CAPM (Capital Asset Pricing Model) usado para determinar el costo del capital propio, en entorno de mercados altamente desarrollados, se puede adaptar en su aplicación a las empresas que carecen de betas bursátiles como son las empresas adventistas y, en vez de ello, usar las betas contables.

3. La proyección de los flujos de caja libre y su crecimiento se pueden abordar desde un enfoque estadístico a partir de la propia información histórica de las empresas adventistas y seleccionando el método con mejor resultado de error estadístico.

Pedro Gonzales Urbina email: pedrog@um.edu.mx 


\section{Pedro Gonzales}

\section{Referencias}

Burton, J. (1998). Revisting the capital asset pricing model. Recuperado en: http://www.stanford. edu/ wfsharpe/art/djam/djam.htm

Casanovas, M., y Bertrán, J., (2013). La financiación de la empresa. España: Editorial Bresca.

Castilla, P. (2011). Valor presente de una perpetuidad. Recuperado en: http://cashflow88.com/ decisiones/VP Perpetuidad_Pedro:Castilla.pdf

Compañía e_Valora (2011). Nociones de valoración de empresas. Recuperado en: https://www. evalora.com/sites/default/files/e-Valora-Manual-de-Valoracion.pdf

Cooper, D., and Chapman, Y., (1987). Risk Analysis for Large Projects. USA: Chichester,John Wiley \& Sons.

Del Álamo V., Alvear L., (2003). Principales métodos de valoración. Price waterhouse Coopers. 2aa ed. / rev. España. FT Prentice Hall.

Dreizzen, D., Yasioja, M., Capomassi, J. y Panelati, M. Conceptos financieros para evaluación de proyectos. Recuperado en: http://materias.fi.uba.ar/7299/PresentacionTPAbrMay08.pdf

Dumrauf, G. (2003). Tendencias en valuación. Buenos Aires, Argentina. Recuperado en: http:// www.ucema.edu.ar/u/gl24/Slides/Tendencias en Valuacion.pdf

Espitia, M., y Gema, A. (2003). Las opciones reales y su influencia en la valoración de empresas. Recuperado en: http://www.dteconz.unizar.es/DT2003-01.pdf

Fernández, P. (2001). Valoración de empresas. Cómo medir y gestionar la creación de valor. Barcelona (España): Ediciones Gestión 2000.

Fernández, P., Cooper, D., y CHAPMAN, Y. (2001). Risk Analysis for Large Projects. USA: Chichester,John Wiley \& Sons,

Forsiyth, J. (2006). Finanzas empresariales: rentabilidad y valor. Lima, Perú: Asociación Gráfica Educativa.

García A. (2006). Un estudio teórico sobre la gestión de riesgos financieros en las empresas. Recuperado en: http://www.monografias.com/trabajos-pdf/gestion-riesgosfinancieros/gestion-riesgos-financieros.pdf

Gitman, L.(1986). Fundamentos de administración financiera. Cuba: Ministerio de Educación Superior.

Polimeni, R., Fabozzi, F., Adelberg, A. y Kole M. (1998). Contabilidad de costos: concepto y aplicaciones para la toma de decisiones gerenciales. (3ra ed.). México: Mc Graw Hill.

Rodríguez, V., Aca, M. (2010). El flujo de efectivo descontado como método de valuación de empresas mexicanas en el período 2001-2007. Revista Contaduría y Administración, 232 (1), 143-172

Saavedra, M. (2007). La valuación de empresas cotizadas en México, mediante la metodología del modelo de flujo de efectivo disponible. México: Contaduría y Administración UNAM $\mathrm{N}^{\circ} 223$, septiembre diciembre.

Thompson M., Antezana J. (2008) Coso de oportunidad. Recuperado en: http://www. promonegocios.net/costos/costos-oportunidad.html

Williams G. (2006). Cómo correr riesgos. Recuperado en: http://todosobrefranquicias.com/ Articulos\%2020Como\%20correr\%20ries gos\%20en \%20los\%20Negocios\%20y\%20 Franquicias.htm 